\title{
Factorised steady states for multi-species mass transfer models
}

\author{
T. Hanney \\ SUPA and School of Physics, University of Edinburgh, Mayfield Road, \\ Edinburgh, EH9 3JZ, UK \\ E-mail: tom.hanney@ed.ac.uk
}

\begin{abstract}
A general class of mass transport models with $Q$ species of conserved mass is considered. The models are defined on a lattice with parallel discrete time update rules. For one-dimensional, totally asymmetric dynamics we derive necessary and sufficient conditions on the mass transfer dynamics under which the steady state factorises. We generalise the model to mass transfer on arbitrary lattices and present sufficient conditions for factorisation. In both cases, explicit results for random sequential update and continuous time limits are given.
\end{abstract}

\section{Introduction}

A wide variety of physical phenomena may be characterised by the stochastic transfer of one or more conserved quantities between regions of space. Examples include traffic flow [1, 2], shaken granular systems [3, sandpile dynamics 4, cluster aggregation and fragmentation [5] and the dynamics of phase separation [6. However, for models of such processes, which are devised on the basis of some putative microscopic dynamics in order to capture the essential elements of these systems, there exists no general prescription to identify the steady state, if indeed it exists at all.

Among the models that have been proposed, particularly well studied examples are the zero-range process (ZRP) [7] [8] and the asymmetric random average process 9. 10. These models involve mass transfer between the sites of a lattice. Chief among the reasons for the widespread interest in these models is that they can be solved exactly in the steady state, a virtue which has been exploited to substantially develop our understanding of jamming transitions and phase separation phenomena. In the framework of zero-range processes, these phenomena may be understood in terms of condensation - the property that a single site may acquire a finite fraction of the total mass in the system. Moreover, the steady state assumes a relatively simple form, namely, a factorised form, in which the steady state is expressed as a product of factors, one factor for each site of the system.

For models with a single conservation law and mass transfer rules which depend only on the details of the departure site, the conditions on the hop rates under which the steady state factorises have now been established rather completely [11. However systems with many conservation laws are also of wide interest [12]. In the soluble cases identified so far, the inclusion of additional conservation laws has been shown to enrich the phase behaviour dramatically and introduces novel mechanisms of condensation 
13. Practical applications of these mass transfer models with multiple conservation laws include shaken polydisperse granular systems 14 and directed networks 15, 16.

It is the purpose of this article to derive conditions on the mass transfer dynamics under which the steady state assumes a factorised form for a very wide class of models with an arbitrary number $Q$ of conservation laws. In section 2 we define a onedimensional, totally asymmetric model with $Q$ species of conserved mass and present the factorised steady state. The proof and the necessary and sufficient conditions under which it holds are given in section 3 The model is defined with discrete-time parallel update, which contains the random sequential update and continuous time limits shown in section 4 The generalisation to a $Q$-species mass transfer model on an arbitrary lattice is presented in section 5 where sufficient conditions for factorisation of the steady state are stated. A number of models which represent applications of the steady states derived here are provided throughout.

\section{2. $Q$-species mass transfer model in one dimension}

The model is defined on a periodic chain containing $L$ sites. $Q$ species of masses reside on the lattice. Associated with each site $i$ is a mass vector $m_{i}$, the elements of which represent the mass of each species $q=1, \ldots, Q$ present at $\bar{i}: \underline{m_{i}}=\left(m_{i}^{(1)}, \ldots, m_{i}^{(Q)}\right)$. The mass of species $q$ at site $i, m_{i}^{(q)}$, is a continuous variable.

The dynamics are defined in discrete time, such that the mass vectors at each site are updated in parallel during each time step, and the mass is transferred to the nearest neighbour site to the right. The mass of species $q$ transferred from site $i$ to site $i+1$ during the time step is $\mu_{i}^{(q)}$. The vector $\underline{\mu}_{i}=\left(\mu_{i}^{(1)}, \ldots, \mu_{i}^{(Q)}\right)$ represents the masses of all species which move from $i$ to $i+1$.

The definition of the model is completed by defining the dynamics. These are specified through the mass transfer probabilities $\psi_{i}\left(\underline{\mu}_{i} \mid \underline{m}_{i}\right)$ which determine the stochastic variables $\mu_{i}^{(q)}$. Hence, the probability of transferring the masses $\underline{\mu}_{i}$ from $i$ to $i+1$ in a time step depends on the mass of each species at the departure site, $\underline{m}_{i}$, and on the site of departure (through the $i$ subscript on $\psi$ ). The dynamics conserve to total mass in the system of each species. Further, the interactions are zero-range - they depend only on details of the site of departure of the mass.

\subsection{Steady state}

The aim is to establish the conditions under which steady state of the model defined above assumes a factorised form. Therefore we look for conditions on the choice of the mass transfer probabilities under which the steady state weight $F\left(\left\{\underline{m}_{i}\right\}\right)$ to observe the system in a configuration $\left\{\underline{m}_{i}\right\}=\underline{m}_{1}, \ldots, \underline{m}_{L}$ is given by

$$
F\left(\left\{\underline{m}_{i}\right\}\right)=\prod_{i=1}^{L} f_{i}\left(\underline{m}_{i}\right),
$$

i.e., one factor for each site of the system. We find a necessary and sufficient condition for factorisation is that one can find functions $v(\underline{\mu})$ and $w_{i}(\underline{m}-\underline{\mu})$ such that the mass transfer probabilities can be expressed in the form

$$
\psi_{i}\left(\underline{\mu}_{i} \mid \underline{m}_{i}\right)=\frac{v\left(\underline{\mu}_{i}\right) w_{i}\left(\underline{m}_{i}-\underline{\mu}_{i}\right)}{\left[v * w_{i}\right]\left(\underline{m}_{i}\right)}
$$


where the $*$ denotes a convolution (see (14)). If the mass transfer probabilities are of the form (2), then the steady state factorises with single-site weights $f_{i}\left(\underline{m}_{i}\right)$ given by

$$
f_{i}\left(\underline{m}_{i}\right)=\left[v * w_{i}\right]\left(\underline{m}_{i}\right) .
$$

\section{Proof of the steady state}

In this section, we turn to the proof of the steady state (1) to (3). The proof follows a similar argument to that given in [17, 18, for the single species case. We write $F\left(\left\{\underline{m}_{i}\right\}, t\right)$ to represent the weight of a configuration at time $t$. For a single update the evolution of $F\left(\left\{\underline{m}_{i}\right\}, t\right)$, under the dynamics defined by the mass transfer probabilities, is described by a master equation which may be written

$$
\begin{aligned}
F\left(\left\{\underline{m}_{i}^{\prime}\right\}, t+1\right)=\prod_{i}^{L}[ & \int \mathrm{d}^{Q} \underline{m}_{i} \int \mathrm{d}^{Q} \underline{\mu}_{i} \psi_{i}\left(\underline{\mu}_{i} \underline{\underline{m}}_{i}\right) \\
& \left.\left.\times \delta^{Q}\left(\underline{\underline{m}}_{i}^{\prime}-\underline{\underline{m}}_{i}+\underline{\mu}_{i}-\underline{\mu}_{i-1}\right)\right)\right] F\left(\left\{\underline{m}_{i}\right\}, t\right),
\end{aligned}
$$

where $\mathrm{d}^{Q} \underline{m}_{i}$ represents an integration over the masses of all the species at site $i$ : $\mathrm{d}^{Q} \underline{m}_{i}=\prod_{q=1}^{Q} \mathrm{~d} m_{i}^{(q)} ;$ similarly, the notation $\delta^{Q}(\underline{x})=\prod_{q=1}^{Q} \delta\left(x^{(q)}\right)$.

In the steady state, $F\left(\left\{\underline{m}_{i}\right\}, t+1\right)=F\left(\left\{\underline{m}_{i}\right\}, t\right) \stackrel{\equiv}{\equiv} F\left(\left\{\underline{m}_{i}\right\}\right)$. Making these replacements in (4) and assuming $F\left(\left\{\underline{m}_{i}\right\}\right)$ is given by a factorised form (10) yields

$$
\begin{aligned}
\prod_{i=1}^{L} f_{i}\left(\underline{m}_{i}^{\prime}\right)=\prod_{i}^{L}\left[\int\right. & \mathrm{d}^{Q} \underline{m}_{i} \int \mathrm{d}^{Q} \underline{\mu}_{i} \psi_{i}\left(\underline{\mu}_{i} \mid \underline{m}_{i}\right) \\
& \left.\times \delta^{Q}\left(\underline{m}_{i}^{\prime}-\underline{m}_{i}+\underline{\mu}_{i}-\underline{\mu}_{i-1}\right) f_{i}\left(\underline{m}_{i}\right)\right] .
\end{aligned}
$$

The aim of the remainder of this section is to find the conditions under which the assumption of factorisation holds, i.e., the conditions under which we can solve (5).

To proceed, we introduce the Laplace transform

$$
g_{i}\left(\underline{s}_{i}\right)=\int_{0}^{\infty} \mathrm{d}^{Q} \underline{m}_{i} \mathrm{e}^{-\underline{s}_{i} \cdot \underline{m}_{i}} f_{i}\left(\underline{m}_{i}\right)
$$

where $\underline{s}_{i}=\left(s_{i}^{(1)}, \ldots, s_{i}^{(Q)}\right)$ and $\underline{s} \cdot \underline{m}=\sum_{q} s^{(q)} m^{(q)}$. Hence

$$
\begin{aligned}
\prod_{i=1}^{L} g_{i}\left(\underline{s}_{i}\right)=\prod_{i}^{L}\left[\int\right. & \mathrm{d}^{Q} \underline{m}_{i} \int \mathrm{d}^{Q} \underline{\mu}_{i} \psi_{i}\left(\underline{\mu}_{i} \mid \underline{m}_{i}\right) \\
& \left.\times \mathrm{e}^{-\underline{s}_{i} \cdot\left(\underline{m}_{i}-\underline{\mu}_{i}+\underline{\mu}_{i-1}\right)} f_{i}\left(\underline{m}_{i}\right)\right] .
\end{aligned}
$$

At this stage, it is convenient to introduce the function $\mathcal{P}_{i}\left(\underline{\mu}_{i}, \underline{m}_{i}-\underline{\mu}_{i}\right)$ defined as

$$
\mathcal{P}_{i}\left(\underline{\mu}_{i}, \underline{m}_{i}-\underline{\mu}_{i}\right)=\psi_{i}\left(\underline{\mu}_{i} \mid \underline{m}_{i}\right) f_{i}\left(\underline{m}_{i}\right),
$$

then, doing the integral over the mass variables for each species, $m_{i}^{(q)}$, and re-ordering the sum in the exponential leads to

$$
\prod_{i=1}^{L} g_{i}\left(\underline{s}_{i}\right)=\prod_{i}^{L}\left[\int \mathrm{d}^{Q} \underline{\mu}_{i} \mathrm{e}^{-\underline{s}_{i} \cdot\left(\underline{m}_{i}-\underline{\mu}_{i}\right)-\underline{s}_{i+1} \cdot \underline{\mu}_{i}} \mathcal{P}_{i}\left(\underline{\mu}_{i}, \underline{m}_{i}-\underline{\mu}_{i}\right)\right] .
$$

Now, the term in the square brackets on the rhs can be identified with the function $X_{i}\left(\underline{s}_{i}, \underline{s}_{i+1}\right)$ :

$$
X_{i}\left(\underline{s}_{i}, \underline{s}_{i+1}\right)=\int \mathrm{d}^{Q} \underline{\mu}_{i} \mathrm{e}^{-\underline{s}_{i} \cdot\left(\underline{m}_{i}-\underline{\mu}_{i}\right)-\underline{s}_{i+1} \underline{\mu}_{i}} \mathcal{P}_{i}\left(\underline{\mu}_{i}, \underline{m}_{i}-\underline{\mu}_{i}\right),
$$


hence, we seek a solution to

$$
\prod_{i=1}^{L} g_{i}\left(\underline{s}_{i}\right)=\prod_{i=1}^{L} X_{i}\left(\underline{s}_{i}, \underline{s}_{i+1}\right)
$$

in order to establish the conditions for factorisation of the steady state. Note that we have only rewritten (5) at this stage - a necessary and sufficient solution of (11) represents a necessary and sufficient condition for factorisation.

By taking the logarithm of (11) and differentiating with respect to $s_{i}$ then $s_{i+1}$, it can be shown [11, 17] that a necessary and sufficient condition to solve (11) is

$$
X_{i}\left(\underline{s}_{i}, \underline{s}_{i+1}\right)=\alpha_{i}\left(\underline{s}_{i}\right) \beta\left(\underline{s}_{i+1}\right),
$$

where the functions $\alpha_{i}(\underline{s})$ and $\beta(\underline{s})$ are to be determined. This implies

$$
g_{i}\left(\underline{s}_{i}\right)=\alpha_{i}\left(\underline{s}_{i}\right) \beta\left(\underline{s}_{i}\right) .
$$

Since this is just a product over Laplace transforms, its inverse (i.e., $f_{i}\left(\underline{m}_{i}\right)$ ) is a convolution

$$
f_{i}(\underline{m})=\left[v * w_{i}\right](\underline{m}) \equiv \int \mathrm{d}^{Q} \underline{\mu} v(\underline{\mu}) w_{i}(\underline{m}-\underline{\mu}),
$$

as given in (3).

The functions $\alpha_{i}(\underline{s})$ and $\beta(\underline{s})$ are determined by

$$
\alpha_{i}\left(\underline{s}_{i}\right)=\int \mathrm{d}^{Q} \underline{\nu} \mathrm{e}^{-\underline{s}_{i} \cdot \underline{\nu}} w_{i}(\underline{\nu}),
$$

where $\underline{\nu}=\underline{m}-\underline{\mu}$, and

$$
\beta\left(\underline{s}_{i}\right)=\int \mathrm{d}^{Q} \underline{\mu} \mathrm{e}^{-\underline{s}_{i} \cdot \underline{\mu}} v(\underline{\mu}) .
$$

By substituting these into (12), and using (10), one can read off

$$
\mathcal{P}_{i}\left(\underline{\mu}_{i}, \underline{m}_{i}-\underline{\mu}_{i}\right)=v\left(\underline{\mu}_{i}\right) w_{i}\left(\underline{m}_{i}-\underline{\mu}\right)
$$

then from the definition of $\mathcal{P}$ given in (8), equation (2) immediately follows.

\section{Continuous time limit}

The discrete time update contains as a special case random sequential update which in turn contains the continuous time limit. The random sequential limit emerges when the mass transport probabilities are proportional to a small time step $d t$ such that at most one mass transfer event on the lattice takes place within a time step. Then the limit $d t \rightarrow 0$ yields continuous time dynamics where the masses are transferred with a rate per unit time. We take the random sequential limit first by redefining

$$
v(\underline{\mu})=\delta^{Q}(\underline{\mu})+x(\underline{\mu}) \mathrm{d} t .
$$

Upon substitution into (3) this yields

$$
f_{i}\left(\underline{m}_{i}\right)=w_{i}\left(\underline{m}_{i}\right) \delta^{Q}(\underline{\mu})+\mathrm{d} t\left[x * w_{i}\right]\left(\underline{m}_{i}\right)+\mathcal{O}\left(\mathrm{d} t^{2}\right),
$$

for the single-site weights, and from (2) it yields

$$
\begin{aligned}
\psi_{i}\left(\underline{\mu} \mid \underline{m}_{i}\right)= & \delta^{Q}(\underline{\mu})-\frac{\mathrm{d} t}{w_{i}\left(\underline{m}_{i}\right)} \delta^{Q}(\underline{\mu})\left[x * w_{i}\right]\left(\underline{m}_{i}\right) \\
& +\mathrm{d} t \frac{x(\underline{\mu}) w_{i}\left(\underline{m}_{i}-\underline{\mu}\right)}{w_{i}\left(\underline{m}_{i}\right)}+\mathcal{O}\left(\mathrm{d} t^{2}\right),
\end{aligned}
$$


for the mass transfer probabilities. In the next step, we take the continuous time limit by taking $d t \rightarrow 0$, hence the single-site weights are

$$
f_{i}(\underline{m})=w_{i}(\underline{m}) \text {, }
$$

and the hop rates are read off as

$$
u_{i}\left(\underline{\mu}^{\prime} \underline{m}_{i}\right)=\frac{x(\underline{\mu}) w_{i}(\underline{m}-\underline{\mu})}{w_{i}(\underline{m})} .
$$

This hop rate represents the rate at which masses $\mu^{(1)}, \ldots, \mu^{(Q)}$ are simultaneously transferred from site $i$ to site $i+1$. If the hop rates can be written in the form (22), the steady state factorises with single-site weights given by (21).

We note that the form (22) implies a constraint on the choice of hop rates. Viewed as a recursion, one can express $w_{i}(\underline{m})$ in terms either $w_{i}(\underline{m}-\underline{\mu})$ or $w_{i}\left(\underline{m}-\underline{\mu}^{\prime}\right)$, before a second application of the recursion yields an expression in terms of $w_{i}\left(\underline{m}-\underline{\mu}-\mu^{\prime}\right)$; this final expression must be the same regardless of the intermediate step, which demands that the hop rates satisfy

$$
\frac{u_{i}(\underline{\mu} \mid \underline{m})}{u_{i}\left(\underline{\mu} \mid \underline{m}-\underline{\mu}^{\prime}\right)}=\frac{u_{i}\left(\underline{\mu}^{\prime} \mid \underline{m}\right)}{u_{i}\left(\underline{\mu}^{\prime} \mid \underline{m}-\underline{\mu}\right)}
$$

for all $i, \underline{m}, \underline{\mu}$ and $\underline{\mu}^{\prime}$. This constraint is not an additional constraint to (22): if the hop rates can be written in the form (22), they automatically satisfy (23). However, it provides an alternative test for factorisation for a given set of hop rates. Moreover, this constraint represents a necessary and sufficient condition for factorisation.

\subsection{Two-species ZRP with disorder}

The ZRP with two species of particles is a discrete-mass model in which single units of mass, either of species 1 or of species 2, are transferred to the nearest neighbour site to the right 19, 20. Here, the model is generalised to include disorder in the hop rates. In the current notation, hop rates for species 1 and species 2 are, respectively,

$$
\begin{aligned}
& u_{i}\left(1,0 \mid m_{i}^{(1)}, m_{i}^{(2)}\right)=\frac{x(1,0) f_{i}\left(m_{i}^{(1)}-1, m_{i}^{(2)}\right)}{f_{i}\left(m_{i}^{(1)}, m_{i}^{(2)}\right)}, \\
& u_{i}\left(0,1 \mid m_{i}^{(1)}, m_{i}^{(2)}\right)=\frac{x(0,1) f_{i}\left(m_{i}^{(1)}, m_{i}^{(2)}-1\right)}{f_{i}\left(m_{i}^{(1)}, m_{i}^{(2)}\right)},
\end{aligned}
$$

which imply the constraint

$$
\frac{u_{i}\left(1,0 \mid m_{i}^{(1)}, m_{i}^{(2)}\right)}{u_{i}\left(1,0 \mid m_{i}^{(1)}, m_{i}^{(2)}-1\right)}=\frac{u_{i}\left(0,1 \mid m_{i}^{(1)}, m_{i}^{(2)}\right)}{u_{i}\left(0,1 \mid m_{i}^{(1)}-1, m_{i}^{(2)}\right)},
$$

The single-site weights, obtained by iterating (2425), are

$$
f_{i}\left(m^{(1)}, m^{(2)}\right)=\prod_{x=1}^{m^{(1)}} u_{i}\left(1,0 \mid x, m^{(2)}\right)^{-1} \prod_{y=1}^{m^{(2)}} u_{i}(0,1 \mid 0, y)^{-1},
$$

having set $f_{i}(0,0)=1$ and absorbed $x(1,0)$ and $x(0,1)$ into the definitions of the hop rates without loss of generality. So, for any given hop rates, 26] represents a necessary and sufficient condition for the steady state to factorise with $f_{i}(\underline{m})$ given by (27). This generalises the results of [19, 20] to the disordered case. 


\subsection{A two-species model}

In this model, again we consider two species of discrete masses. Now, the number of particles of a species which hops depends on the number of particles of the other species

at the departure site. Hence, we consider the following rates: if $m_{i}^{(1)}>m_{i}^{(2)}>0$, then $m_{i}^{(2)}$ particles of species 1 hop with a rate

$$
u\left(m_{i}^{(2)}, 0 \mid m_{i}^{(1)}, m_{i}^{(2)}\right)=\frac{f\left(m_{i}^{(1)}-m_{i}^{(2)}, m_{i}^{(2)}\right)}{f\left(m_{i}^{(1)}, m_{i}^{(2)}\right)} ;
$$

if $m_{i}^{(2)}>m_{i}^{(1)}>0$, then $m_{i}^{(1)}$ particles of species 2 hop with a rate

$$
u\left(0, m_{i}^{(1)} \mid m_{i}^{(1)}, m_{i}^{(2)}\right)=\frac{f\left(m_{i}^{(1)}, m_{i}^{(2)}-m_{i}^{(1)}\right)}{f\left(m_{i}^{(1)}, m_{i}^{(2)}\right)} ;
$$

if $m_{i}^{(1)}=m_{i}^{(2)}=m>0$ then, with equal probability, either $m$ particles of species 1 hop with a rate

$$
u(m, 0 \mid m, m)=\frac{f(0, m)}{f(m, m)},
$$

or $m$ particles of species 2 hop with a rate

$$
u(0, m \mid m, m)=\frac{f(m, 0)}{f(m, m)} .
$$

If only a single species is present at a site, single particles hop with rates

$$
u(1,0 \mid m, 0)=\frac{f(m-1,0)}{f(m, 0)}, \quad u(0,1 \mid 0, n)=\frac{f(0, n-1)}{f(0, n)} .
$$

A simple choice for the rates is $u(n, 0 \mid m, n)=(1+n / m)^{b}$ for $m \geq n>0$ and $u(0, m \mid m, n)=(1+m / n)^{b}$ for $n \geq m>0$; the single-site weights for these rates are given by $f(m, n)=(m+n)^{-b}$. Though the model presented here is new, singlesite weights of this form have been considered in 16] where it was shown that a condensation transition occurs above a critical particle density for $b>3$. Ordinarily, the two species condense at completely independent sites; in the present model however, an exclusion interaction exists between the condensates: by considering the hop rates in the limit of large $n$ and $m$, it is clear that if both species condense at the same time, they do so at randomly located sites subject to the condition that the condensates of each species do not occupy the same site.

\section{Generalisation to hypercubic and arbitrary lattices}

The models considered above can be generalised to $Q$-species mass-transfer models on arbitrary lattices. The derivation of the conditions for factorisation can also be generalised along the lines of [1]. Though this derivation is straightforward, its simplicity is rather obscured under complicated notation; therefore we present in this section the results without providing details. We also provide concrete examples of models with factorised steady states in order to illustrate the utility of the results. 


\subsection{Generalised model}

Again, we begin with discrete time dynamics whereby each site is updated in parallel. The new ingredient is that $Q$ fixed sets of directed links now connect certain pairs of sites - each set is associated with a particular species and mass of any species can only be transferred along associated directed links.

To define the update rules, we define a mass transfer matrix for each species $q$ with elements $\mu_{i j}^{(q)}$. These elements represent the mass of species $q$ which is transferred from site $i$ to site $j$ during a time step. The vector $\underline{\mu}_{i j}=\left(\mu_{i j}^{(1)}, \ldots, \mu_{i j}^{(Q)}\right)$ represents the masses of all species which move from $i$ to $j$; if there is no directed link for species $q$ pointing from $i$ to $j$, then $\mu_{i j}^{(q)}=0$ identically. Also, $\mu_{i i}^{(q)}$ is in general nonzero and represents the mass of species $q$ at site $i$ that does not hop during the time-step.

The elements $\mu_{i j}^{(q)}$ which are not set to zero for all times (i.e., those which are accompanied by a directed link pointing from $i$ to $j$ ) are determined by the mass transfer probabilities $\psi_{i}\left(\left\{\underline{\mu}_{i j}\right\} \mid \underline{m}_{i}\right)$. The set $\left\{\underline{\mu}_{i j}\right\}$ contains all sites $j$ to which $i$ is connected by a directed link for at least one species and includes site $i$ itself. Thus it specifies the mass transferred for each and every species which can hop from $i$ to $j$.

As before, the dynamics conserve the total mass of each species in the system. The sum over the elements in a row of the mass transfer matrix for species $q$ is equal to the mass of species $q$ at the site before the update: $\sum_{j} \mu_{i j}^{(q)}=m_{i}^{(q)}$. Similarly, the sum over column elements represents the mass $m_{i}^{\prime(q)}$ of species $q$ at $i$ after the update: $\sum_{j} \mu_{j i}^{(q)}=m_{i}^{\prime(q)}$.

\subsection{Conditions under which the steady state factorises}

Using the multi-species procedure presented in section 3 and following the technique outlined in [11] for one species of mass on an arbitrary lattice, one can establish conditions on the generalised mass transfer probabilities under which the steady state assumes the factorised form (11).

We find a sufficient condition for factorisation (but no longer necessary in general) is that one can find functions $v_{i j}\left(\underline{\mu}_{i j}\right)$ such that the mass transfer probabilities can be expressed in the form

$$
\psi_{i}\left(\left\{\underline{\mu}_{i j}\right\} \mid \underline{m}_{i}\right)=\frac{\prod_{j} v_{i j}\left(\underline{\mu}_{i j}\right)}{\left[\prod_{* j} v_{j i}\right]\left(\underline{m}_{i}\right)},
$$

subject to the constraint $\sum_{j} \mu_{i j}^{(q)}=m_{i}^{(q)}$ for all species $q$. Here, the product in the numerator is over sites $j$ which have a directed link coming from site $i$ for at least one species; the product in the denominator is over all sites which have at least one directed link pointing into $i$ and the $*$ denotes a convolution form. If the mass transfer probabilities are of the form (33), then the steady state factorises with single-site weights $f_{i}\left(\underline{m}_{i}\right)$ given by

$$
f_{i}\left(\underline{m}_{i}\right)=\left[\prod_{* j} v_{j i}\right]\left(\underline{m}_{i}\right),
$$

which again is a multiple convolution over those sites which have a directed link into $i$ for at least one species. 
A second condition for factorisation is that the functions $v_{i j}\left(\underline{\mu}_{i j}\right)$ satisfy

$$
\left[\prod_{* j} v_{i j}\right]\left(\underline{m}_{i}\right)=\left[\prod_{* j} v_{j i}\right]\left(\underline{m}_{i}\right),
$$

which emerges as a constraint on the geometry of the underlying lattice. This constraint will typically limit the choices allowed for the dynamics on inhomogeneous lattices. On homogeneous lattices, there exist two particular classes of dynamics for which the constraint is always satisfied: (i) detailed balance - in cases where the dynamics are symmetric, i.e., $v_{i j}\left(\underline{\mu}_{i j}\right)=v_{j i}\left(\underline{\mu}_{j i}\right)$ the constraint (35) is always satisfied; (ii) pair-wise detailed balance - the constraint (35) is always satisfied in cases where, for each species, there exists a site $i$ with a link into $j$ for every link from $j$ to another site $k$, such that $v_{i j}\left(\underline{\mu}_{i j}\right)=v_{j k}\left(\underline{\mu}_{j k}\right)$. A simple example is asymmetric nearest neighbour mass transport in one dimension, where transfer to $i$ from $i-1$ can be paired with transfer from $i$ to $i+1$. There are many more ways to satisfy the constraint, some of which are discussed below, but dynamics which fall into either of the above two categories are sufficiently prevalent in mass transport models that we wish to emphasise that the constraint is automatically fulfilled in these two cases.

The general conditions under which (33) represents a necessary condition seem difficult to formulate [11]: though necessary for totally asymmetric mass transport in one dimension, as outlined above, it is not necessary for symmetric mass transport. It is also necessary for mass transfer on the complete graph.

\subsection{Continuous time limit}

The random sequential then continuous time limits are obtained as before, by redefining

$$
v_{i j}\left(\underline{\mu}_{i j}\right)=\delta^{Q}\left(\underline{\mu}_{i j}\right)+x_{i j}\left(\underline{\mu}_{i j}\right) \mathrm{d} t .
$$

In the limit $d t \rightarrow 0$, the single-site weights assume the form

$$
f_{i}\left(\underline{m}_{i}\right)=v_{i i}\left(\underline{m}_{i}\right) \text {, }
$$

and hop rates are obtained as

$$
u_{i j}\left(\underline{\mu} \mid \underline{m}_{i}\right)=\frac{x_{i j}(\underline{\mu}) v_{i i}\left(\underline{m}_{i}-\underline{\mu}\right)}{v_{i i}\left(\underline{m}_{i}\right)} .
$$

This hop rate represents the rate at which masses $\mu^{(1)}, \ldots, \mu^{(Q)}$ are simultaneously transferred from site $i$ to site $j$. Finally, in the continuous time limit, the constraint acquires the form

$$
\sum_{j \neq i}\left[x_{i j} * v_{i i}\right]\left(\underline{m}_{i}\right)=\sum_{j \neq i}\left[x_{j i} * v_{i i}\right]\left(\underline{m}_{i}\right) .
$$

By taking the Laplace transform, cancelling common factors then inverting Laplace transform back, the constraint can be written

$$
\sum_{j \neq i} x_{i j}(\underline{\mu})=\sum_{j \neq i} x_{j i}(\underline{\mu}) .
$$

Therefore, if the hop rates can be written in the form (38), and provided the constraint (40) is satisfied, the steady state factorises with single-site weights given by (37). 


\subsection{Generalised Q-species mass-transfer model in one dimension}

In the case where each particle species is transferred between nearest neighbour sites on the same one-dimensional lattice, the constraint (40) implies

$$
x_{i, i+1}(\underline{\mu})+x_{i, i-1}(\underline{\mu})=x_{i+1, i}(\underline{\mu})+x_{i-1, i}(\underline{\mu}),
$$

and the general solution has been obtained in [21]; this solution must be used in order to allow disorder in the asymmetry. Here, we take the pair-wise balance solution

$$
\begin{aligned}
& x_{i, i+1}(\underline{\mu})=p(\underline{\mu}), \\
& x_{i+1, i}(\underline{\mu})=q(\underline{\mu}),
\end{aligned}
$$

for all $i$. Hence the steady state factorises if the hop rates can be written in the form

$$
\begin{aligned}
& u_{i, i+1}\left(\underline{\mu} \mid \underline{m}_{i}\right)=\frac{p(\underline{\mu}) f_{i}\left(\underline{m}_{i}-\underline{\mu}\right)}{f_{i}\left(\underline{m}_{i}\right)}, \\
& u_{i, i-1}\left(\underline{\mu}^{\prime} \underline{m}_{i}\right)=\frac{q(\underline{\mu}) f_{i}\left(\underline{m}_{i}-\underline{\mu}\right)}{f_{i}\left(\underline{m}_{i}\right)},
\end{aligned}
$$

for all $i$. Note that some disorder is still incorporated through the site dependence in $v_{i i}(\underline{m})$ : the disorder is in the departure rates rather than the asymmetry.

The two-species, totally asymmetric limit $q(\mu)=0$ with transfer of single units of discrete mass recovers the limit discussed in section 4.1

\subsection{Generalised Q-species mass transfer on a hypercubic lattice}

Here, we consider homogeneous, symmetric dynamics on a hypercubic lattice: masses $\underline{\mu}=\left(\mu^{(1)}, \ldots, \mu^{(Q)}\right)$ are transferred to a randomly selected nearest neighbour site with $\overline{\text { a }}$ rate $u(\mu \mid \underline{m})$.

In this case, the dynamics satisfy detailed balance so the constraint (40) is easily seen to be satisfied for $x_{i j}(\mu)$ independent of $i$ and $j$. The sufficient condition for factorisation then is that the hop rates satisfy the constraint

$$
\frac{u\left(\underline{\mu} \mid \underline{m}_{i}\right)}{u\left(\underline{\mu} \mid \underline{m}_{i}-\underline{\mu}^{\prime}\right)}=\frac{u\left(\underline{\mu}^{\prime} \mid \underline{m}_{i}\right)}{u\left(\underline{\mu}^{\prime} \mid \underline{m}_{i}-\underline{\mu}\right)},
$$

in which case the single-site weights are determined from

$$
u\left(\underline{\mu} \mid \underline{m}_{i}\right)=\frac{f\left(\underline{m}_{i}-\underline{\mu}\right)}{f\left(\underline{m_{i}}\right)},
$$

where the constant factors $x(\mu)$ have been absorbed into $u$. Thus, since the rates are homogeneous, the single-site weights are independent of $i$. This generalises the results of 22] to an arbitrary number of species of particles. Generalisations to include disorder or to partial asymmetry along the lines of the section 5.4 are straightforward.

\subsection{Two-species ZRP on an arbitrary lattice}

In this section, we consider the two-species ZRP again, but this time on an arbitrary lattice. We consider the case where both species hop on the same underlying lattice, with hop rates from site $i$ to site $j$ given by

$$
\begin{aligned}
& u_{i j}\left(1,0 \mid m_{i}^{(1)}, m_{i}^{(2)}\right)=y_{i j} \alpha_{i}^{(1)}\left(m_{i}^{(1)}, m_{i}^{(2)}\right), \\
& u_{i j}\left(0,1 \mid m_{i}^{(1)}, m_{i}^{(2)}\right)=y_{i j} \alpha_{i}^{(2)}\left(m_{i}^{(1)}, m_{i}^{(2)}\right),
\end{aligned}
$$


for species 1 and 2 , respectively. Here, $\alpha_{i}^{(q)}\left(m_{i}^{(1)}, m_{i}^{(2)}\right)$ represents the total departure rate of particles of species $q$ from site $i$, for $q=1,2 ; y_{i j}$ represents the probability that a particle is transferred from site $i$ to site $j$. The same probabilities are used for both species because they are both moving on the same underlying lattice (though this is straightforward to generalise).

The choices

$$
x_{i j}(\underline{\mu})=y_{i j} p_{i}
$$

for $\underline{\mu}=(1,0)$ or $(0,1)$, and

$$
\begin{aligned}
& \alpha_{i}^{(1)}\left(m_{i}^{(1)}, m_{i}^{(2)}\right)=\frac{p_{i} f_{i}\left(m^{(1)}-1, m^{(2)}\right)}{f_{i}\left(m^{(1)}, m^{(2)}\right)}, \\
& \alpha_{i}^{(2)}\left(m_{i}^{(1)}, m_{i}^{(2)}\right)=\frac{p_{i} f_{i}\left(m^{(1)}, m^{(2)}-1\right)}{f_{i}\left(m^{(1)}, m^{(2)}\right)},
\end{aligned}
$$

are of the form (38) from which the single-site weights are obtained as

$$
f_{i}\left(m^{(1)}, m^{(2)}\right)=p_{i}^{m_{i}^{(1)}+m_{i}^{(2)}} \prod_{x=1}^{m^{(1)}} \alpha_{i}^{(1)}\left(x, m^{(2)}\right)^{-1} \prod_{y=1}^{m^{(2)}} \alpha_{i}^{(2)}(0, y)^{-1}
$$

From the constraint (40), the $p_{i}$ 's are determined by

$$
p_{i}=\sum_{j \neq i} y_{j i} p_{j}
$$

which is just the solution for the steady state of a single random walker moving on the lattice with hopping probabilities defined by the $y_{i j}$ (c.f. (41)).

Again the two equations (51, 52) imply a constraint on the possible choices of hop rates for which the steady state still factorises:

$$
\frac{\alpha_{i}^{(1)}\left(m_{i}^{(1)}, m_{i}^{(2)}\right)}{\alpha_{i}^{(2)}\left(m_{i}^{(1)}, m_{i}^{(2)}-1\right)}=\frac{\alpha_{i}^{(2)}\left(m_{i}^{(1)}, m_{i}^{(2)}\right)}{\alpha_{i}^{(2)}\left(m_{i}^{(1)}-1, m_{i}^{(2)}\right)} .
$$

If this equation is satisfied, a model with rates (48, 49) factorises with single-site weights given by (53) with the $p_{i}$ 's determined by (54) - thus it is the $p_{i}$ 's which contain the information about the structure of the underlying lattice.

\section{Conclusion}

We have found conditions for factorised steady states applicable to a very wide class of models. The results have been exploited to write down the exact steady states for several models which generalise previously solved cases. The interplay between conservation laws, geometry, disorder and the nature of interactions can all be explored within the framework of the steady states derived here and merit further investigation.

As intimated in the introduction, condensation is of particular interest in mass transfer models. For homogeneous two-species systems, the general conditions for condensation are well understood at the level of the steady state 23. However the models considered here are substantially more general, therefore it is of interest to establish whether new condensed phases emerge. Even for the two species model considered in section 4.2] which has a steady state in the class considered in [23], the exclusion interaction between condensates seems to be a property of the dynamics which has not been observed in previously studied models. 
Moreover, the coarsening dynamics in two-species models have been studied using heuristic, quasi-static scaling arguments 24, and are far richer than their singlespecies counterparts. It is reasonable to suppose that the wider class of models solved here will further enrich the phenomenology of the coarsening dynamics.

Finally, the question of the structure of steady states beyond the factorised form remains open [25] but must be addressed in order to understand behaviour observed in non-factorisable models [26].

\section{Acknowledgments}

The author thanks Martin Evans for useful discussions and acknowledges the SUPA and the EPSRC for support under programme grant GR/S10377/01.

\section{References}

[1] Chowdhury D, Santen L and Schadschneider A, 2000 Physics Reports 329199

[2] O'Loan OJ, Evans MR and Cates ME, 1998 Phys. Rev. E 581404

[3] van der Meer D, van der Weele K and Lohse D, 2002 Phys. Rev. Lett. 88174302

[4] Jain K, 2005 Phys. Rev. E 72017105

[5] Majumdar SN, Krishnamurthy S and Barma M, 1998 Phys. Rev. Lett. 813691

[6] Kafri Y, Levine E, Mukamel D, Schütz GM and Török J, 2002 Phys. Rev. Lett. 89035702

[7] Spitzer F, 1970 Adv. Math. 5246

[8] Evans MR and Hanney T, 2005 J. Phys. A 38 R195

[9] Krug J and Garcia J, 2000 J. Stat. Phys. 9931

[10] Rajesh R and Majumdar SN, 2000 J. Stat. Phys. 99943

[11] Evans MR, Majumdar SN and Zia RKP, 2006 J. Phys. A 394859

[12] Schütz GM, 2003 J. Phys. A 36 R339

[13] Hanney T and Evans MR, 2004 Phys. Rev. E 69016107

[14] Mikkelsen R, van der Meer D, van der Weele K and Lohse D, 2002 Phys. Rev. Lett. 89214301

[15] Dorogovtsev SN, Mendes JFF and Samukhin AN, 2003 Nucl. Phys. B 666396

[16] Angel AG, Hanney T, and Evans MR, 2006 Phys. Rev. E 73016105

[17] Evans MR, Majumdar SN and Zia RKP, 2004 J. Phys. A 37 L275

[18] Zia RKP, Evans MR and Majumdar SN, 2004 J. Stat. Mech.:Theor. Exp. L10001

[19] Großkinsky S and Spohn H, 2003 Bull. Braz. Math. Soc. 34489

[20] Evans MR and Hanney T, 2003 J. Phys. A 36 L441

[21] Derrida B, 1983 J. Stat. Phys. 31433

[22] Greenblatt RL and Lebowitz JL, 2006 J. Phys. A 391565

[23] Großkinsky S, to be published

[24] Großkinsky S and Hanney T, 2005 Phys. Rev. E 72016129

[25] Evans MR, Hanney T and Majumdar SN, 2006 Phys. Rev. Lett. 97010602

[26] Godrèche C, 2006 J. Phys. A 399055 